\title{
Genetic relationships of poplar species in section Tacamahaca based on cpDNA and ISSR
}

\author{
Ke Chen*, Xiuqing Jia, Peng Ren and Jianqing Liu \\ School of Life Science and Engineering, Southwest University of Science and Technology, Mianyang, \\ Sichuan 621010, P. R. China. \\ Accepted 15 August, 2011
}

\begin{abstract}
A molecular investigation on the species of Populus belonging to section Tacamahaca was undertaken with the aim of inferring genetic relationships within the section. The ISSR and cpDNA RFLP-based UPGMA analyses were performed for seven Populus species, with 50 specimens representing 10 accessions from southeastern Qinghai-Tibetan Plateau, China. The results provided molecular evidence suggesting that Populus schneideri is highly genetically similar to Populus kangdingensis, as also indicated by previous studies with respect to their morphological characters. Similarly, the combination of cpSSR and nuclear SSR analyses showed that Populus trinervis and Populus simonii have a close genetic affinity, indicating their closed sister relationships. Although Populus yunnanensis is distinct from the other species based on nuclear ISSR analysis; it formed a clade with $P$. simonii and $P$. trinervis. This reflects similar maternal phylogeny among the three species. In addition, the cluster analyses clearly showed a close relationship among accessions of the same species and suggested monophyly in Populus przewalskii and Populus cathayana. Our results demonstrated that the combination of cpDNA-RFLP and ISSR molecular markers enables a clear estimation of genetic relationships among Populus species and they provided information for preliminary conclusions that will be helpful in guiding future research.
\end{abstract}

Key words: Populus, section Tacamahaca, genetic relationships.

\section{INTRODUCTION}

The genus Populus L. is a member of the Salicaceae family and is subdivided into six sections. They are widely distributed in the forests of temperate and cold regions of the Northern hemisphere (Brawdshaw et al., 2000; Cervera et al., 2005). Populus are major supplier of industrial wood and fiber in many countries and have become the model of choice for molecular genetics and genomics research on forest trees because of their fast growth rates, easy vegetative propagation, adaptability to a variety of ecological sites and amenability to genetic transformation (Fossati et al., 2005; Xu et al., 2009). The southeastern Qinghai-Tibetan Plateau is abundant in poplar gene resources including 17 species and 15 varieties identified. With 11 species, it alone comprise

${ }^{\star}$ Corresponding author. E-mail: Michael_chenke@yahoo.cn. Tel: +86-816-6383210. Fax: +86-816-6383210.
$46 \%$ of the 24 endemic poplar species of the section Tacamahaca occurring in China (Yu et al., 2003). In fact, southeastern Qinghai-Tibetan Plateau is regarded as the natural distribution and variation center for poplar species of the section Tacamahaca in China. The vertical distribution of these poplar trees ranges from 1,300 to 4,300 $\mathrm{m}$ above sea level (a.s.l.). Even under unfavourable climatic conditions at high altitudes and with a short growth season, some species (that is Populus kangdingesis) stand out due to their high vitality, remarkable growth potential and great adaptability. However, little is currently known about the majority of the poplar species occurring in this region. Although Populus is the model tree species for biological research, information on interspecific phylogeninetic relationships in the genus is rather limited (Fussi et al., 2010). The placement of species within a section has traditionally been based on morphological and reproductive characters, as well as interspecific crossability. However, 
Table 1. The investigated Populus specimens, all of the section Tacamahaca, and their natural altitude distribution, codes and origin.

\begin{tabular}{|c|c|c|c|c|}
\hline Species & Common name & Natural altitude distribution $(\mathrm{m})$ & Codes & Origin \\
\hline P. kangdingensis, C. Wang et Tung & Kangding poplar & $2,100 \sim 4,300$ & $\mathrm{KD}$ & Kangding, Sichuan \\
\hline P. schneideri, Rehd. & - & $2,650 \sim 3,900$ & $\mathrm{DC}$ & Daocheng, Sichuan \\
\hline P. przewalskii, Maxim & - & $2,900 \sim 3,400$ & $\begin{array}{l}A B \\
X N\end{array}$ & $\begin{array}{l}\text { Aba, Sichuan } \\
\text { Xining, Qinghai }\end{array}$ \\
\hline P. cathayana, Rehd & Green poplar & $1,900 \sim 3,000$ & $\begin{array}{l}J Z \\
P W \\
H Y\end{array}$ & $\begin{array}{l}\text { Jiuzhai, Sichuan } \\
\text { Pingwu, Sichuan } \\
\text { Hanyuan, Sichuan }\end{array}$ \\
\hline P. trinervis, C. Wang et Tung & Threevein poplar & $1,500 \sim 2,500$ & KDSM & Kangding, Sichuan \\
\hline P. simonii, Carr. & Simon poplar & $1,250 \sim 2,400$ & KDXY & Kangding, Sichuan \\
\hline P. yunnanensis, Dode & Yunnan poplar & $1,300 \sim 3,000$ & LD & Luding, Sichuan \\
\hline
\end{tabular}

classical taxonomic analysis based on morphological characteristics has proven to be very difficult because of wide intraspecific variability, high natural crossability among members of the genus, and the convergent morphology shown by hybrids and their parental species (Cervera et al., 2005).

Since the late 1980s, several molecular marker approaches have been successfully used in a number of poplar species for the fingerprinting and identification of clones and the determination of their interrelationships: isozymes, RAPD, ISSR, AFLP and SNP markers (Liu and Furnier 1993; Rahman and Rajora 2002; Gao et al., 2006; Olson et al., 2010), mitochondrial and chloroplast restriction site analyses (Barrett et al., 1993; Heinze 1998), and sequence analyses primarily on the nuclear ITS and on the noncoding chloroplast regions (Hamzeh and Dayanandan, 2004). In addition, the combined use of cpDNA (maternally inherited marker) and nuclear DNA (biparentally inherited marker) data is crucial when aiming to gain a more comprehensive understanding of the evolutionary history of plants (Hamzeh and Dayanandan, 2004). In the present study, cpDNA and nuclear ISSR markers were used to differentiate seven closely related Populus species belonging to the section Tacahamaca and to estimate their genetic relationships.

\section{MATERIALS AND METHODS}

\section{Plant materials and DNA extraction}

Seven species of the genus Populus in section Tacamahaca from southeastern Qinghai-Tibetan Plateau, China were analyzed in this study. Details of the collection sites, natural altitude distributions and accession codes are given in Table 1. DNA was extracted from $0.5 \mathrm{~g}$ fresh young leaf material from each individual following a modified protocol of Castiglione et al. (1993). Concentrations and quality of DNA was checked by a DNA-Protein instrument (BioRAD). In our study, the DNA mixing strategy was used for the ISSR and cpDNA PCR-RFLP assay. For this template-mixing strategy, equal amounts of working solution DNAs from each five individuals of the same accession were pooled as template DNA prior to the PCR reaction.

\section{ISSR amplification}

Initially, 29 ISSR markers which were used to construct a genetic map of the Populus genome (Yin et al., 2002) and 30 ISSR markers developed by the Biotechnology Laboratory, University of British Columbia (UBC, Vancouver, Canada) were tested. Based on the clarity and reproducibility of the band patterns, 11 primers were selected from the 59 primers tested for the further studies (Table 2). PCR amplification was performed in a $20-\mu$ l reaction volume containing $2.0 \mu \mathrm{l}$ of the $10 \times$ reaction buffer (TaKaRa, Dalian, $\mathrm{Mg}^{2+}$ free), $1.8 \mathrm{mM} \mathrm{MgCl} 2$ buffer, $150 \mu \mathrm{M}$ dNTP (Promega), $0.25 \mu \mathrm{M}$ primer, $1.0 \mathrm{U}$ Taq polymerase (TaKaRa, Dalian) and $25 \mathrm{ng}$ of genomic DNA. The mixture was overlaid with mineral oil and subjected to PCR on a GeneAmp® PCR System 9700 (Perkin Elmer Corp., Norwalk, CT, USA) programmed for an initial step of 5 min at $95^{\circ} \mathrm{C}$, followed by 40 cycles of $1 \mathrm{~min}$ at $94^{\circ} \mathrm{C}, 1 \mathrm{~min}$ at annealing temperature (Table 2) and $2 \mathrm{~min}$ at $72^{\circ} \mathrm{C}$, with a final extension step of $10 \mathrm{~min}$ at $72^{\circ} \mathrm{C}$. PCR products were analyzed on $1.8 \%$ agarose gels and stained with ethidium bromide. The gel images were recorded and the band sizes were quantified using a Gel Doc 2000 system (Bio-RAD). Each PCR reaction was performed at least twice to ensure the reproducibility.

\section{PCR-amplification of cpDNA and mtDNA non-coding regions}

Five sets of chloroplast primers and four sets of mitochondrial primers were used to amplify non-coding regions of cpDNA and mtDNA shown in Table 3 (Demesure et al., 1995). The amplification reactions were performed in a volume of $25 \mu$ l containing reaction buffer, $200 \mu \mathrm{M}$ of dNTP (Promega), $0.2 \mu \mathrm{M}$ of each primer, $1.0 \mathrm{U}$ of Taq polymerase (TaKaRa) and $20 \sim 50 \mathrm{ng}$ of DNA. The PCR 
Table 2. The nucleotide sequences of the eight selected ISSR primers and the number of bands scored.

\begin{tabular}{ccccc}
\hline Primer & Primer sequences $\left(\mathbf{5}^{\prime}-\mathbf{3}^{\prime}\right)$ & Annealing temp. $\left({ }^{\circ} \mathbf{C}\right)$ & No. of alleles & PIC value \\
\hline IP-2 & $(\mathrm{AC})_{8} \mathrm{C}$ & 54 & 12 & 0.454 \\
IP-4 & $(\mathrm{GA})_{8} \mathrm{CTT}$ & 54 & 8 & 0.455 \\
24 & $(\mathrm{AC})_{8} \mathrm{TC}$ & 55 & 16 & 0.486 \\
33 & $(\mathrm{AG})_{8} \mathrm{AT}$ & 53 & 17 & 0.398 \\
177 & $(\mathrm{AC})_{8} \mathrm{YG}$ & 52 & 9 & 0.432 \\
807 & $(\mathrm{AG})_{8} \mathrm{~T}$ & 55 & 11 & 0.497 \\
808 & $(\mathrm{AG})_{8} \mathrm{C}$ & 56 & 13 & 0.401 \\
809 & $(\mathrm{AG})_{8} \mathrm{G}$ & 57 & 12 & 0.382 \\
826 & $(\mathrm{AC})_{8} \mathrm{C}$ & 53 & 11 & 0.392 \\
834 & $(\mathrm{AG})_{8} \mathrm{YT}$ & 53 & 7 & 0.334 \\
848 & $(\mathrm{CA})_{8} \mathrm{RG}$ & 55 & 14 & 0.422 \\
\hline
\end{tabular}

Table 3. The cpDNA and mtDNA primer pair sequences and annealing temperatures.

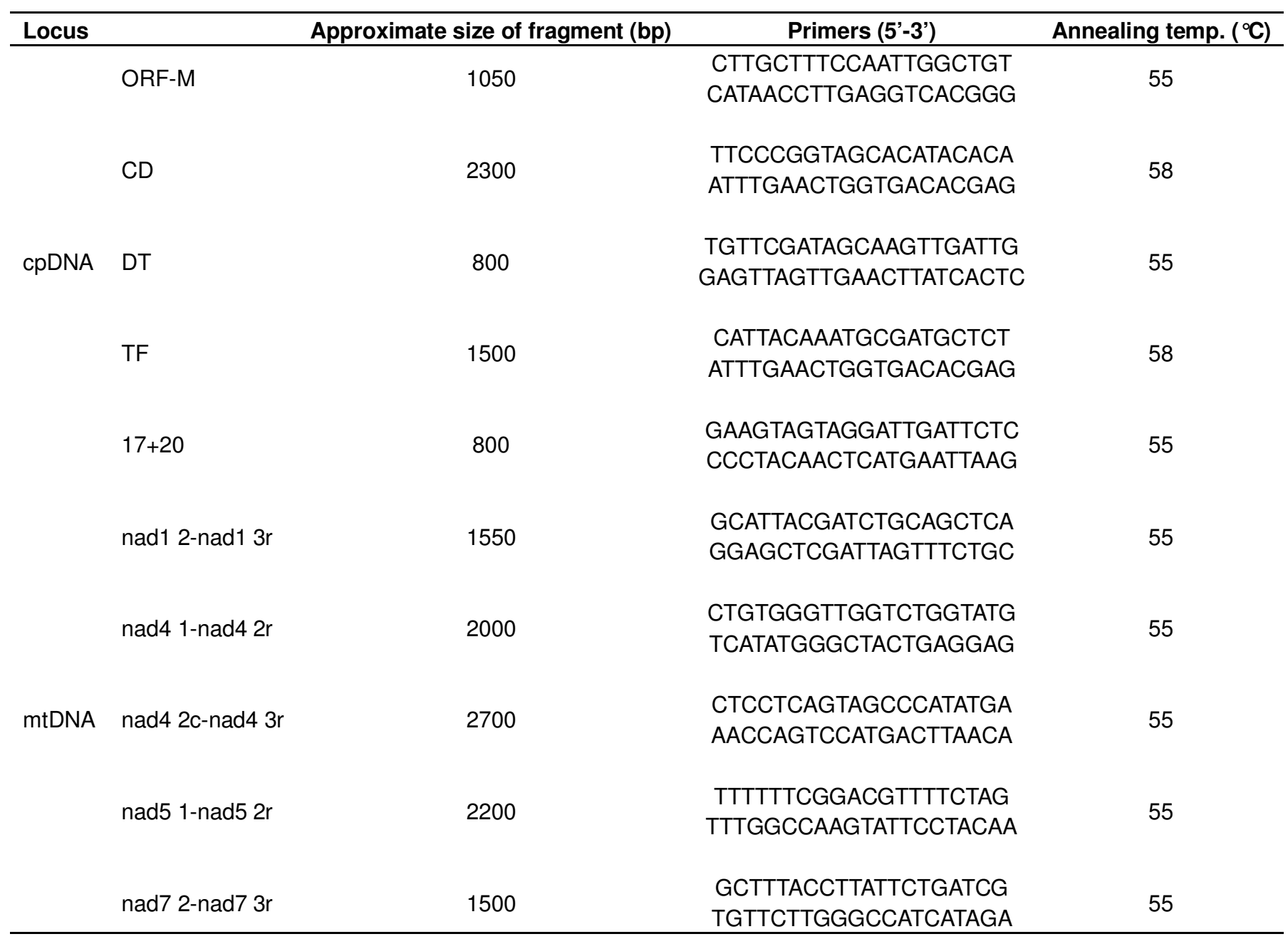

conditions for DT, CD and TF were as follows: $95^{\circ} \mathrm{C}$ for $15 \mathrm{~min}$, followed by 40 cycles of $93^{\circ} \mathrm{C}$ for $45 \mathrm{~s}, 55^{\circ} \mathrm{C}$ or $58^{\circ} \mathrm{C}$ for $45 \mathrm{~s}$ and $72^{\circ} \mathrm{C}$ for $2 \mathrm{~min}$ followed by $72^{\circ} \mathrm{C}$ for $10 \mathrm{~min}$. For ORF-M, there was an initial step of $95^{\circ} \mathrm{C}$ for 15 min followed by 30 cycles of $95^{\circ} \mathrm{C}$ for $50 \mathrm{~s}, 55^{\circ} \mathrm{C}$ for $50 \mathrm{~s}$ and $72^{\circ} \mathrm{C}$ for $1 \mathrm{~min}$ followed by $72^{\circ} \mathrm{C}$ for $10 \mathrm{~min}$.
The PCR conditions for $17+20$ were $95^{\circ} \mathrm{C}$ for 15 min followed by 20 cycles of $95^{\circ} \mathrm{C}$ for $50 \mathrm{~s}, 58^{\circ} \mathrm{C}$ for $50 \mathrm{~s}$ with a reduction of $0.5^{\circ} \mathrm{C}$ per cycle and $72^{\circ} \mathrm{C}$ for $1 \mathrm{~min} 45 \mathrm{~s}$ followed by further 20 cycles of $95^{\circ} \mathrm{C}$ for $50 \mathrm{~s}, 50^{\circ} \mathrm{C}$ for $50 \mathrm{~s}$ and $72^{\circ} \mathrm{C}$ for $1 \mathrm{~min} 45 \mathrm{~s}$ followed by $72^{\circ} \mathrm{C}$ for $10 \mathrm{~min}$. For mtDNA primers: $5 \mathrm{~min}$ at $94^{\circ} \mathrm{C}$; 35 cycles of 1 


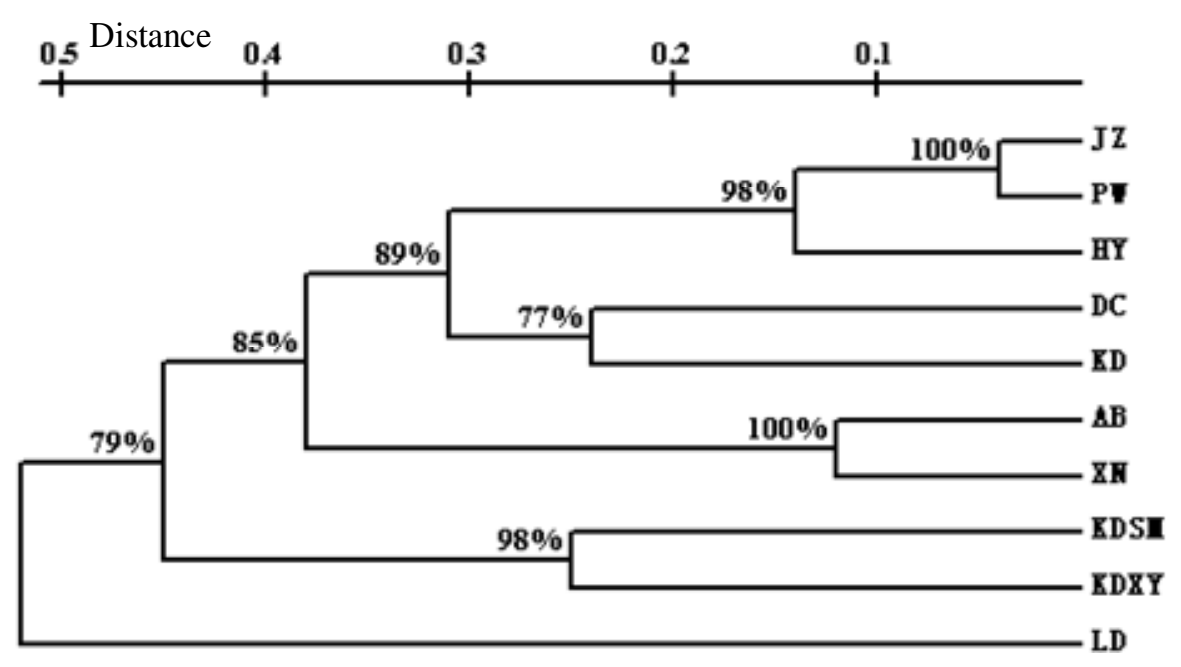

Figure 1. UPGMA dendrogram obtained for the Populus Section Tacamahaca by nuclear ISSR analysis using the Nei's index. Bootstrap values above 50\% obtained from 1000 replicate analyses are shown.

$\min$ at $94^{\circ} \mathrm{C}, 1 \mathrm{~min}$ annealing at $55^{\circ} \mathrm{C}$ and $2 \min$ extension at $72^{\circ} \mathrm{C}$; followed by $72^{\circ} \mathrm{C}$ for $10 \mathrm{~min}$. The success of each PCR reaction was verified by electrophoresing $2 \mu$ of the reaction products with $1 \%$ agarose gel. The PCR-amplified DNA fragments were digested using four restriction enzymes (Hinfl, Taql, EcoRI and BamHI; New England Biolabs, Ltd.) for the cpDNA regions and mtDNA region. The restriction digests were visualized on $2 \%$ agarose gel stained with ethidium bromide.

\section{Data analysis}

For the ISSR analysis, the fragment size scored ranged from 200 to $1500 \mathrm{bp}$. Weak bands were excluded from final data analysis. Since ISSRs were interpreted as dominant markers, the bands were scored as diallelic characters either as 1 (present) or 0 (absent). A pair-wise similarity matrix was calculated using the simple matching coefficient. This similarity matrix was employed to construct a dendrogram by UPGMA or neighbour joining (NJ) using the TREECON 1.3 b software. Branch support was estimated with 500 bootstrap replicates. A cophenetic matrix was derived from the similarity matrix to test the goodness of fit of the clusters by comparing the two matrices using the Mantel matrix correspondence test in the MXCOMP program of the NTSYS-pc package. In addition, the program DCENTER, EIGEN and MOD3D of NTSYS-pc 2.1p were used to perform a principle coordinates (PCO) analysis for the nuclear ISSR character matrix. For cpDNA and mtDNA PCR-RFLP data analysis, the digested DNA fragments were scored by presence (1) or absence (0) for each Populus accession. To facilitate comparison between different marker systems, the data matrices were analyzed by the same computer programs as described earlier for ISSR data analysis.

\section{RESULTS}

In the current experiment, consistent results were obtained across at least two DNA mixture template replicates for the three marker systems with over $97 \%$ of scorable fragments reproducible for ISSR and $100 \%$ for cpDNA and mtDNA-RFLP. Very faint fragments were not reproducible, thus such fragments were not scored in this study. ISSR amplifications using 11 primers generated a total of 130 reliable fragments from the template-mixing patterns of the ten Populus accessions. The size of these fragments ranged between 200 to $1500 \mathrm{bp}$ (Table 2). The minimum and maximum number of fragments generated per primer in all the accessions studied were 7 (primer 834) and 17 (primer 33), respectively (Table 2), with an average of 11.8 fragments per primer. Of the total 130 scorable fragments, $119(91.5 \%)$ bands were polymorphic among accessions and only 11 bands were simultaneously present in the 10 Populus species. 22 bands (approximately 16.9\%) were found to be speciesspecific. The polymorphic information content (PIC) for each ISSR primers varied from 0.333 to 0.493 with a mean of 0.423 (Table 2). The primer 807 was found to be the most informative for species differentiation, whereas primer 834 was the least informative. The PCR-RFLP survey of the chloroplast DNA from the 10 Populus accessions investigated revealed 52 polymorphic fragments, which together allowed the identification of 9 haplotypes. Only the two accessions of Populus prezewaskii shared the same haplotype. Four non-coding regions of mtDNA were also amplified with the universal primers. PCR amplification of these regions revealed no length polymorphism among accessions. These PCR products were further digested using four restriction enzymes, but neither site variation nor length polymorphism was revealed among the 10 accessions studied. Thus, no mtDNA-RFLP analysis was considered necessary for further investigation.

The genetic relationships among the 10 accessions of Populus were analyzed by cophenetic cluster methods (Figure 1). The ISSR phenogram had very high cophenetic correlation coefficient $(r=0.94)$, indicating excellent fits of the similarity matrices to the resulting 


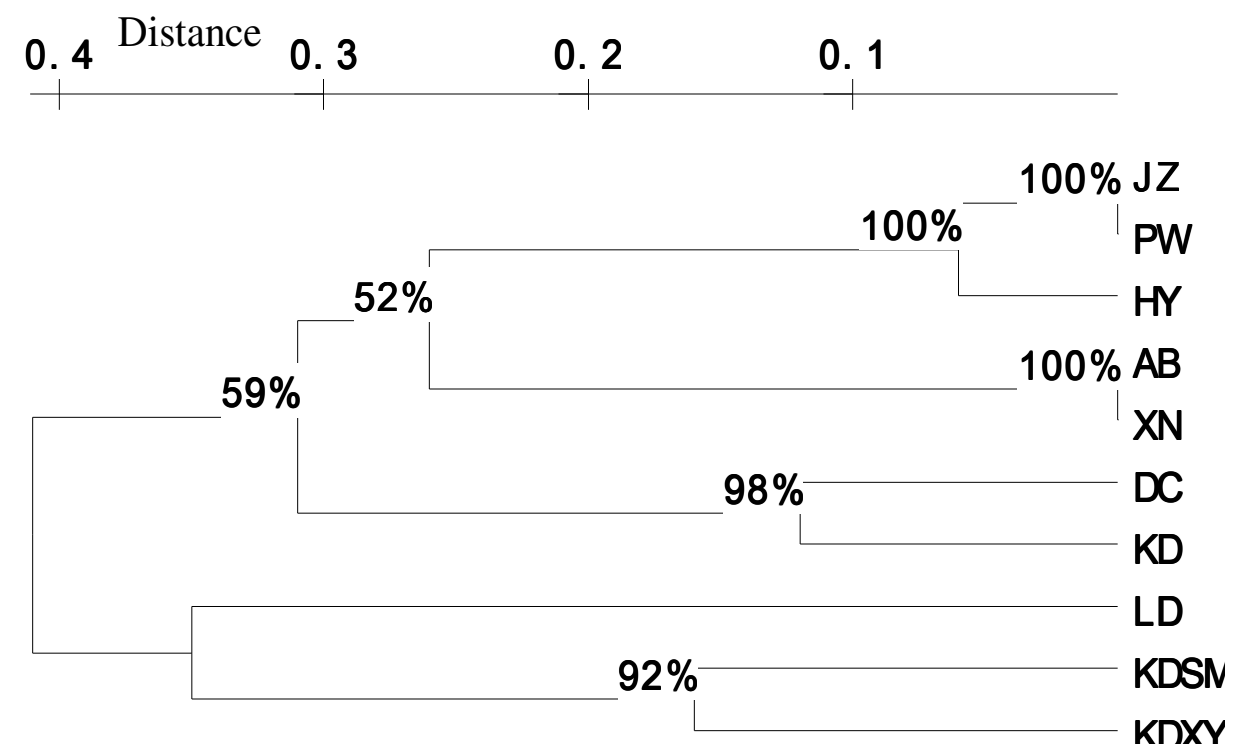

Figure 2. UPGMA dendrogram obtained by cpDNA-RFLP analysis using the Nei and Li (1979) index for the Populus section Tacamahaca. Bootstrap values above $50 \%$ obtained from 1000 replicate analyses are shown.

phenogram while that with cpDNA-RFLP was lower $(r=$ 0.84). However, the topologies of the ISSR and cpDNA phenograms were similar to each other by inspection. Comparing the cpDNA-based cluster tree (Figure 2) with the ISSR-based tree (Figure 1), similar relationships were found. Firstly, the accessions from same Populus species clustered together with high bootstrap value support, that is accessions of $P$. przewalskii and Populus cathayana. Within the $P$. cathayana group, the accession HY was distinct from the accessions JZ and PW with high bootstrapvalue support. Moreover, the close relationships between KD and DC were confirmed, and the two same species were then grouped with $P$. cathayana and $P$. przewalskii. In addition, KDSM and KDXY formed one group with high bootstrap support in both cluster trees indicating their closed sister relationships. However, the major difference between the ISSR and cpDNA trees was the position of Populus yunnanensis which formed a clade with KDSM and KDXY based on a shared cpDNA fragment length polymorphism with low bootstrap value (below 50\%). Additionally, the relationships among the Populus species were further illustrated by the results of a principal coordinate analysis based on the nuclear ISSR character matrix. The first three coordinates accounted for $28.1,24.1$ and $10.3 \%$ of the total variance, respectively and distinguished similar groupings of cpDNA-based tree, which support the placement of $P$. yunannensis with KDSM and KDXY.

The cophenetic matrices computed from chloroplast and nuclear marker-based tree matrices and compared with the original similarity data showed significant correlations of 94.3 and $92.6 \%$, respectively, revealing in both cases a good fit for the cluster analyses performed.

\section{DISCUSSION}

The main objective of the present study was to assess the genetic relationships among the Populus species belonging to the section Tacahamaca on the basis of cpDNA-RFLP and ISSR markers. A striking feature of poplars is the occurrence of interspecific hybrids. In phylogenetic studies conducted on groups in which hybridization between lineages has played a substantial role in their evolution (Smith, 1988), the combined use of cpDNA and nuclear DNA markers is crucial when aiming to acquire a comprehensive understanding of the evolutionary history. ISSR and cPDNA-RFLP markers were proved to be the efficient and inexpensive way to provide molecular data. They have been used successfully in determining genetic relationships and for DNA fingerprinting (Huang, 2000). Tranditionally, Populus species have been grouped within their respective sections based primarily on their interspecific crossability and morphological similarities. The most debates were mainly existed in the species identification in Section Tacamahaca and Aigerios. In present study, all the 7 species of Populus could be distinguished by ISSR and cpDNA-RFLP, respectively, which laid a solid foundation. Since $P$. cathayana, Populus kangdingensis, $P$. schneideri, and $P$. prezewalskii clustered into one group or one clade especially in cpDNA analysis, one can conclude that these species are highly genetically related. Within this group, the highest genetic similarities were found between $P$. kangdingensis and $P$. schneideri. The species $P$. schneideri, which occurs in southeastern Qinghai-Tibetan Plateau at the altitudes of 2,500 4,000 $\mathrm{m}$ a.s.I., is morphologically similar to $P$. cathayana. 
However, unlike $P$. cathayana which has glabrous branchlets and leaves, $P$. schneideri usually has pilose branchlets and leaves, which are common characteristics also in $P$. kangdingensis. In addition genetically closely alike $P$. schneideri and $P$. kangdingensis are both unique to western Sichuan and they share similar altitude and habitat requirements. Thus, the high interspecific ISSR genetic similarity values (data not shown) observed between the $P$. kangdingensis and $P$. schneideri, together with the ISSR and cpDNA analyses, partially corroborate previous suggestion (based on morphological classification) that $P$. schneideri was a variety of $P$. kangdingensis.

Additional molecular, morphological and other analyses with a larger sample size would be required. Another group of highly related species include Populus trinervis and Populus simonii. The high genetic similarities between the two species are consistent with their morphological characters and habitat requirements. Both the two species is the long ovoid capsule, while the other species of the section Tacamahaca usually have ovoid capsules. $P$. trinervis and $P$. simonii are adapted to dry and thermic valley habitats and both are distributed at low altitudes. Although $P$. trinervis and $P$. simonii showed high ISSR and cpDNA similarities, their accessions formed distinct separate groups. Thus, additional molecular, morphological and other analyses with larger sample sizes would be required to corroborate their relationships. The status of $P$. yunnanensis has been unclear. The cluster analysis based on ISSR markers clearly distinct $P$. yunnanensis from the other Populus species. However, these results are not consistent with those reported earlier that $P$. yunnanensis was grouped with $P$. simonii based on AFLP analysis (Cervera et al., 2005). $P$. yunnanensis may share the same chloroplast ancestor with $P$. simonii and $P$. trinervis as indicated by their identical cpDNA analysis (Figure 2). This may reflect their similar maternal phylogeny. Hence, the position of $P$. yunnanensis remains unclear and the analysis of a larger number of accessions is required to clarify its relationship with other Tacamahaca species, especially with $P$. simonii. Mitochondrial PCR-RFLPs showed no site or length polymorphism in the present study. This indicates that the slow evolutionary rate of the mitochondrial genome makes mtDNA PCR-RFLPs unsuitable for resolving phylogenetic relationships at the intrageneric level. In comparison with the high level of restriction site polymorphism detected in cpDNA for the same set of species, this study again provides evidence supporting that the nucleotide substitution rate of mtDNA in plants is much lower than that of cpDNA (Palmer, 1992). In conclusion, the present study showed the pattern of genetic interrelationships and intraspecific variability among the native poplar species in southeastern Qinghai-Tibetan Plateau. Consequently, the new knowledge supplies information for understanding the evolution of Populus from the section Tacamahaca and it will help to guide future research.

Our results demonstrated that the combination of cpDNA and ISSR molecular markers can be practical when monitoring a defined but yet limited set of genetic changes that may have occurred during the evolution process among Populus. However, the range of Populus accessions and species included in this study is limited. Therefore, a more extensive survey of Populus specimens in the section Tacamahaca, based on a wide range of markers covering both non-coding and coding regions including data based on for example AFLPs, DNA sequencing and isozymes would enable us to obtain a more complete view of the genetic variation, interrelationships and evolution among Populus species.

\section{ACKNOWLEDGEMENTS}

The research was supported by Youth Foundation of Sichuan Province Department of Education Fund (No. 09ZB093), the Doctoral Foundation of Southwest University of Science and Technology (08ZX7105) and Laboratory Foundation of Southwest University of Science and Technology (10XNKF32).

\section{REFERENCES}

Barret JW, Rajora OP, Yeh FCH, Dancik BP (1993). Mitochondrial DNA variation and genetic relationships of Populus species. Genome, 36: 87-93.

Brawdshaw HD, Ceulemans R, Davis J, Stettler R (2000). Emerging model systems in plant Biology: Poplar (Populus) as a model forest tree. J. Plant Growth Regul., 19: 306-313.

Castiglione S, Wang G, Damiani G, Bandi C, Bisoffi S, Sala, F (1993). RAPD fingerprints for identification and for taxonomic studies of elite poplar (Populus spp) clones. Theor. Appl. Genet., 87: 54-59.

Cervera MT, Storme V, Soto A, Ivens BM, van Montagu, Rajora OP, Boerjan W (2005). Intraspecific and interspecific genetic and phylogenetic relationships in the genus Populus based on AFLP markers, Theor. Appl. Genet., 111: 1440-1456.

Fossati T, Zapelli I, Bisoffi S, Micheletti A, Vietto L, Sala F, Castiglione S (2005). Genetic relationships and clonal identity in a collection of commercially relevant poplar cultivars assessed by AFLP and SSR. Tree Genet. Genomes., 1: 11-19.

Fussi B, Lexer C, Heinze B (2010). Phylogeography of Populus alba (L.) and Populus temula (L.) in Central Europe: secondary contact and hybridization during recolonisation from disconnected refugia. Tree Genet. Genomes., 6: 439-450.

Gao JM, Zhang SG, Qi LW, Zhang Y, Wang CG, Song WQ (2006). ISSR and AFLP identification and genetic relationships of Chinese elite accessions from the genus Populus. Ann. For. Sci., 63: 1-8.

Hamzeh M, Dayanandan S (2004). Phylogeny of Populus (Salicaceae) based on nucleotide sequences of chloroplast trnT-trnF region and nuclear rDNA. AM. J. Bot., 91(9): 1398-1408

Heinze B (1998). PCR-based chloroplast DNA assay for the identification of native Populus nigra and introduced poplar hybrids in Europe. Forest Genet., 5:31-38

Huang JC, Sun M (2000). Genetic diversity and relationships of sweetpotato and its wild relatives in Ipomoea series Batatas (Convolvulaceae) as revealed by inter-simple sequence repeat (ISSR) and restriction analysis of chloroplast DNA. Theor. Appl. Genet., 100(7):1050-1060.

Liu Z, Furnier GR (1993). Comparison of allozyme, RFLP, and RAPD markers for revealing genetic variation within and between trembling 
aspen and bigtooth aspen. Theor. Appl. Genet., 87: 97-105

Olson MS, Robertson AL, Takebayashi N, Silim S, Schroeder WR, Tiffin $P$ (2010). Nucleotide diversity and linkage disequilibrium in balsam poplar (Populus balsamifera). New Phyt., 186:526-536.

Palmer JD (1992). Mitochondrial DNA in plant systematics: application and limitations. In: Soltis PS, Soltis DE, Doyle JJ (eds) Molecular systematics of plants. Chapman and Hall New York, pp. 36-49.

Rahman MH, Rajora OP (2002). Microsatellite DNA fingerprinting, differentiation, and genetic relationships of clones, cultivars, and varieties of six poplar species from three sections of the genus Populus. Genome, 45: 1083-1094.

Smith RL (1988). Phylogenetic of Populus L. (Salicaceae) based on restriction site fragment analysis of cpDNA. M.S. thesis, Universtity of
Wisconsin, Madison, Wisconsin, USA.

Xu MQ, Zhou XD, Piao CG (2009). Populus cultivation-clones in different cultivated area and its diseases in China. J. For. Res., 22(5): 705-714.

Yin TM, Zhang XY, Huang MR, Wang MX, Qiang Z, Tu S, Zhu L, Wu R (2002). Molecular linkage maps of the Populus genome. Genome, 45: $541-555$

Yu SQ, Liu J, Fu DR, Liu DJ, Liu YQ (2003). Characteristics of Tacamahaca genes in the Western Sichuan Plateau. J. Zhejiang For. College, 20(1): 27-31. (in Chinese) 